*Manuscript in press in Current Opinion in Behavioral Sciences (COBEHA)

\title{
Creativity comes in waves: An EEG-focused exploration of the creative brain.
}

\author{
Carl E. Stevens Jr. \& Darya L. Zabelina
}

\author{
University of Arkansas \\ Department of Psychological Science \\ 480 Campus Drive \\ 216 Memorial Hall \\ Fayetteville, AR 72701
}

Declarations of interest: none.

Correspondence concerning this article should be addressed to Darya L. Zabelina, Department of Psychological Science, 480 Campus Drive, Fayetteville, AR 72701. Ph: 479-575-5813. E:

dlzabeli@uark.edu. 


\begin{abstract}
Electroencephalographic (EEG) methodology in creativity research has been remarkably fruitful, establishing the potential of EEG to illuminate complex and transient creativity-related neural processes. Here we synthesize recent advances in the field, highlighting empirical work on creativity as divergent thinking, remote associations, musical creativity, and visual imagery. There is a general consensus that alpha-band activity plays a key role in the creative process, though other frequency bands, such as theta and gamma also serve critical functions. We further discuss evidence for electrical stimulation (tDCS and tACS) as a tool for improvements in creative thinking. EEG's high temporal resolution is supremely suitable for studying creative cognition, and studies continuing to yield new and exciting evidence regarding the local and global neural processes underlying creativity.

Keywords: electroencephalography (EEG); event-related potentials (ERP); transcranial directcurrent stimulation (tDCS); creativity; divergent thinking; remote associations; musical creativity; visual imagery
\end{abstract}




\section{Creativity comes in waves: An EEG-focused exploration of the creative brain.}

\section{Introduction}

Electroencephalography (EEG) has been used for decades, and despite a proliferation in the use of tools for measuring brain activity that depend on more recent technological advances (e.g., functional magnetic resonance imaging [fMRI], magnetoencephalography [MEG]), it remains a highly valuable method [1]). While the use of EEG in creativity research is not historically common, its capacity to deliver supremely high temporal resolution data, including information about temporally coherent and phase-locked network activity, should make it a go-to method for assessing the fast-moving and complex processes of creativity. With respect to sample rate, EEG is far superior to fMRI (typically, one image every two seconds), allowing insights that are not possible with fMRI. Additionally, given the physical constraints required by tools such as fMRI, EEG is often a more appropriate choice for ecologically valid creativity tasks. EEG administration may also be a more tolerable process than fMRI for certain populations (e.g., children, individuals with heightened sensory sensitivity, and those who fear confined spaces). For all these reasons (not to mention time and cost), we consider EEG a tool of primary importance in creativity research.

Here we first provide a brief overview of the EEG frequency bands, and the event-related potentials (ERPs). We then synthesize recent empirical work on the EEG-focused exploration of the creative brain. Namely, we highlight recent studies on creativity as divergent thinking, creativity as remote associations, musical creativity, and creativity as visual imagery. We conclude with a section on the neuromodulation for the creative brain, and offer general conclusions. 


\section{Review of EEG Frequency Bands}

EEG is a non-invasive method for measuring the distribution of voltages across the scalp that are created by the synchronous activity of large populations of neurons $[2,3]$. While these signals are very small ( $\mu \mathrm{V}$ level), their measurement is facilitated by highly sensitive equipment. The downside of this sensitivity is that it opens the door for environmental noise and movement artifacts in the data, although various filters, movement-specific channels, and motionless participants, help combat these noise sources. EEG has relatively poor localization of the signal's source due to the infinite set of possible neural activation patterns that could create a given EEG measurement, however, it has excellent temporal resolution, with possible sample rates in the thousands of Hertz.

Like colors in the visible range of the electromagnetic spectrum, EEG frequency bands occupy specific regions of the overall oscillatory spectrum. The rate at which oscillations occur defines frequency (measured in Hertz), or cycles per second, i.e., completion of one up and down wave pattern. From lowest to highest, EEG bands are categorized as delta (0.5-4 Hz), theta (4-8 $\mathrm{Hz})$, alpha $(8-13 \mathrm{~Hz})$, beta $(13-30 \mathrm{~Hz})$, and gamma $(30+\mathrm{Hz})$. While the higher bands oscillate more frequently, the amplitudes of lower waves are greater. This results in a typical left-to-right downward slope in graphs of spectral density, which shows the power of oscillations at points along the overall spectrum over a given time range (Figure 1). EEG analyses typically use methods such as Fourier transforms to create such time-frequency outputs [2].

Although the borders are informed by nearly a century of research [1], assigning meaning to activity in the different EEG bands is a complex matter. Phenomena most commonly associated with each band include: Delta - deep sleep [1]; theta - error monitoring, cognitive control [2]; alpha - eye closed, relaxation, internally-directed attention, network inhibition [2, 4]; 
beta - being awake, affective and cognitive processing [5]; and gamma - active processing of sensory information, perceptual binding $[2,6]$.

\section{Review of ERPs}

In contrast to time-frequency-based EEG analysis, ERP analysis is focused on aspects of brief perturbances in EEG signals that occur in response to experimenter-defined events. As such, the time range of interest is typically from the point of stimulus onset to the end of the elicited ERP wave. In creativity research, this means that ERP analysis is useful for examining relatively immediate processes, in relation to specific events in the creative thinking process, while time-frequency EEG analysis is better suited for examining states or processes with longer time courses. The peaks and troughs of event-related voltage waves are measured and are typically averaged across subjects, after which between-condition and/or between-subject comparisons can be made [7]. ERP components, or specific time points in the waveform, are of interest to researchers for reasons that depend on the nature of the task. Components are historically named for the number of milliseconds after stimulus onset at which they occur, and whether the wave is positive $(\mathrm{P})$ or negative $(\mathrm{N})$ at that timepoint. Commonly assessed components and their meanings include: P50 - sensory gating [8]; N200 - oddball effect [9]; $\mathrm{N} 2 \mathrm{pc}$ - visual attentional selection [10]; P3a (frontal) - task-related attention [11]; P3b (temporo-parietal) - attention and memory [11]; and N400 - semantic meaning/congruity [12].

\section{Creativity as Divergent Thinking}

EEG and ERP are common methodologies for examining more traditional subfields of cognitive psychology, including attention and memory. Until fairly recently, however, cognitive processes such as creative thinking have not benefited from these methodologies, with the exception of pioneering work in the 1970s $[13,14]$. Fortunately, in recent years there has been a 
re-emergence of interest in using electrophysiology methods for investigating creative cognition, making significant strides towards our understanding of various features of creativity. We highlight these recent findings in the present manuscript.

Creativity has traditionally been defined as the ability to generate ideas that are novel and useful [15], with an undefined set of possible ideas. Divergent thinking (DT) tests assess this ability, and have been used for decades to examine creative idea generation [16]. The Alternate Uses Task (AUT) is one such measure, requiring participants to generate novel uses for familiar objects, with responses scored for the number of uses generated (fluency), and the uniqueness of responses (originality).

The most consistently observed finding using EEG suggests that alpha-band activity is linked with better performance on DT tasks $[17,18]$ (Figure 2a). For example, imposing greater creativity demands in the AUT resulted in higher task-related alpha power (TRP; pre-stimulus interval minus idea generation interval) in frontal and parietal regions [19]. In a similar task, alpha power was greatest at the beginning of creative idea generation, decreased, and then again increased just prior to responding [20]. Individual differences studies have similarly pointed to higher alpha-band power in more, versus less creative people, as assessed by DT tests [21]. Further, people who perform better on DT tests were able to sustain increased alpha in frontal and parietal regions for longer durations [22]. Given that increased alpha is considered to reflect a reduction in externally-oriented attention in favor of more internally-oriented attention [17], these findings may indicate that divergent thinkers are better able to continually exclude interfering external stimuli for the sake of generating creative responses. Indeed, attention appears to play a key role in creativity-related alpha fluctuations. Externally-oriented attention strongly involves visual processing, and has been found to decrease alpha activity during 
divergent thinking [23]. Conversely, when attention is internally oriented, alpha power increases, and merely closing one's eyes has been shown to improve DT performance [24].

In addition to increases in the alpha power during divergent thinking, decreases in delta and theta bands have also been reported. Widespread decreased delta power, for example, was found by examining differences in signals for divergent versus convergent thinking [25]. In a study in which participants provided captions for an assortment of unusual scenes displayed on a computer screen, decreased mid-frontal theta power was found to be associated with higher creativity scores, as rated by naïve participants [26] (Figure 2b). This study also reported that increased frontal-occipital theta functional connectivity was associated with increased creativity. As low frequency ranges are generally suggested to support long-range oscillatory communication, while high frequency ranges support more local communication [27], this finding indicates that creativity may be the result of long-range oscillatory communication among regions that sub-serve creative functions. Theta band activity in frontal regions has also been associated with increased cognitive control [28], which was shown to play a key role in divergent thinking. Indeed, people who performed better on DT tasks engaged cognitive control processes more strongly when an unexpected attentional switch was required, as indicated by a larger N2 difference between the frequent and rare targets in an Oddball task [29].

Considering that frequency ranges outside of the alpha band appear to be linked with divergent thinking, and the relevance of attention and cognitive control for creativity [30, 31 , 32], it will be worthwhile for future creativity studies to examine the EEG frequency bands associated with these functions. 


\section{Creativity as Remote Associations}

Creativity has also been considered as the ability to connect relatively remote concepts or ideas, with the facility for making novel associations signaling greater creativity [33]. The Compound Remote Associates (CRA) task [34] was designed to examine this ability by presenting people with three seemingly unrelated words (e.g., pine, crab, sauce), and asking them to name a word that forms a common compound word or phrase with all three words (here the solution word is apple: pineapple, crabapple, apple sauce). There are at least two ways of solving CRA problems: via insight - the so-called Aha! moment that comes suddenly and with little ability to report on the processing that enabled the solution, or via a deliberate, trial-anderror analytical approach [35].

A number of studies have identified specific patterns of brain activity associated with insightful and analytical CRA solutions. For example, alpha power was found to increase in the right parietal region when participants generated solutions with insight, compared to when they merely recognized the answers based on cues [36]. The location of this activity is important, as the parietal cortex is essential for sensory integration [37]. Increased alpha in this area could thus be interpreted as a suppression of sensory information in service of internally-oriented creative processes. Increased alpha when individuals are preparing to solve CRA problems via insight was initially discovered by Jung-Beeman and colleagues [38] (Figure 3a), and consequently replicated in other studies $[35,38]$. Specifically, just prior to the arrival in conscious awareness of a solution reached via insight (versus via analysis), an increase in alpha-band activity (10 Hz) is seen over the occipital cortex [38]. Alpha-band activity is immediately followed by a burst of high frequency gamma-band activity originating in the right temporal lobe. While alpha-band activity is typically the primary finding in creativity research, the gamma activity is particularly 
interesting because (1) gamma oscillations may provide the means for binding features of objects as they emerge into conscious awareness [6], and (2) gamma has been associated with sensory and memory processes, including top-down and bottom-up information matching [39], thus suggesting that insight problem solving may involve the sub-conscious association of information, and the subsequent binding of that information into a percept. Further, the propensity for insightful or analytical thinking can be predicted from resting-state EEG weeks in advance. Using the CRA task, it was found that participants with the propensity to solve anagrams and CRA problems via insight (vs. analysis) showed increased alpha- and theta-band activity over the left temporal lobe, as determined through the localization-improving technique known as a surface-Laplacian transformation [40] (Figure 3b). It was suggested that inhibition of activity in the left temporal lobe, as indicated by increased alpha activity, may allow close associates to be suppressed, and more remote associations to be facilitated. This interpretation depends, in part, on the role of theta oscillations in long-distance integration processes [42]. The findings are in line with current understanding of the attentional demands of creative idea generation and insight, which propose that inhibition of certain regions, particularly in the left hemisphere, allow for "remoter" associates to be selected, as opposed to more local ones [36, 43, 44].

Similar to divergent thinking, top-down, solving problems via insight is linked with internally-oriented attention, as opposed to the more bottom-up, externally-oriented mode of attention found during non-insight problem-solving [44]. Additionally, people whose attention is less internally-oriented at rest, as demonstrated by reduced gamma-band synchronization over the central regions of the default mode network (DMN), tend to be more creative [45]. In summary, neural signatures of insight have been repeatedly differentiated from analytical 
problem-solving using EEG, opening the door for further exploration of the underlying functional differences, and methods designed to promote either mode.

\section{Musical Creativity}

Music is pervasive in our daily lives and has a strong emotional component through which we can connect with the artist and one another [47]. We are so attuned to it that sounds not intended as music can be perceived as such under the right circumstances [48]. As a result, the neural mechanisms of music production and perception are a growing area of research.

Numerous studies have explored the neural mechanisms of music perception in professional musicians via EEG methodology. For example, by use of a technique called multifractal detrended cross-correlation analysis (MFDXA), it was found that when professional musicians imagined music, as opposed to only hearing it, EEG cross-correlations between frontal and occipital regions were greater [48]. Another recent study examined how classically and improvisationally trained musicians responded to hearing short chord progressions with one chord "out of place" (a "functional deviant") [49]. Improvisationally (compared to classically) trained musicians had more discriminable N2c and P3b ERPs in response to deviant chords, indicating both a greater sense of violation of musical expectation, and a stronger sense that the violation required a response. Improvisationally trained musicians also responded faster and were better able to discern deviants, perhaps indicating an effect of training on the brain. It cannot be ruled out, however, that the improvisors sought such training because their brains were already well-suited for it, and that similar effects would not have been found prior to the start of their training.

Interestingly, in a similar study that included improvisors, classical musicians, and nonmusicians, non-musicians demonstrated a greater preference for high-, compared to medium- and 
low-expectation chord progressions. In contrast, improvisors preferred medium expectation chords, and had no preference between high and low expectation [50]. Improvisors also showed increased P3b responses, and a faster return to baseline after deviant chords, compared to classical and non-musicians. In combination, these studies indicate that improvisational training promotes better discernment of the "substitutability" of chords, increased preference for unconventional changes, and faster responses when such changes are encountered.

With respect to music production, compared to musicians who had no formal improvisation training, jazz musicians showed increased frontal alpha-band TRP during improvisation, compared to when they simply heard or played back melodies as instructed [51] (Figure 4). Further, right frontal upper alpha-band activity was associated with higher quality of improvised performances in improvisationally-trained musicians. As with DT tests, increased alpha during periods of creative production likely indicates an inward orienting of attention. Increased alpha activity may therefore be the mechanism by which improvisational jazz musicians perform better on DT tasks than musicians from classical and folk genres [52], as performing jazz music tends to require internally-oriented attention to continuously generate and evaluate musical ideas [53]. The extent to which improvisational training can create long-term, and perhaps domain-general improvements in creativity, is worthy of exploration.

\section{Creativity as Visual Mental Imagery}

Visual mental imagery, defined as an experience of sensory information without a direct external stimulus, relies heavily on the visual cortex $[54,55]$. A recent meta-analysis found that, in contrast to divergent thinking, visual mental imagery was accompanied with decreased alpha, compared to baseline, although alpha power in high-creative groups tended to be greater than for low-creative groups, despite the task-related decreases in both groups [56]. Alpha power was 
also found to decrease in parietal and occipital regions when performing the Picture Completion Task, indicating elevated visual processing demands [57]. Interestingly, when the same participants mentally elaborated on their drawings, alpha power in these regions was greater than during the initial creation phase, perhaps reflecting an increase in internally-oriented attentional demands. Further, this increase in frontal alpha power in the elaboration stage was associated with better creative performance. In another study, compared to non-artists, graphic arts students demonstrated greater increases in spectral coherence among more brain regions, and in more frequency bands (including alpha, beta, and gamma), in increasingly demanding visual imagery tasks [58]. This effect was accompanied by similarly greater decreases among other regions in response to increased task difficulty, indicating alternate processing strategies for highly demanding visual tasks in graphic artists, compared to non-artists.

In a study that used ERPs to study whether mental imagery creates neural adaptation effects (reduced response to repeated stimuli) similar to those observed for visual stimuli, participants either imagined a famous face, or actually viewed it [59]. They were then presented with either the same face or an object (the test stimuli). In both cases, N170/VPP complex responses (thought to be related to face processing) [60] were highly sensitive to face test stimuli and exhibited the same timing effects; these effects, however, were not in the same direction for the two conditions. In the viewing condition, N170/VPP amplitude was suppressed (expected adaptation), while in the mental visualization condition, N170/VPP was increased. This finding is interpreted as indicative of a bottom-up effect for perceptual (viewing) adaptation, and a topdown effect for mental visualization, due in large part to working memory engagement and memory reactivation. In a more recent study, mental imagery of faces again primed the N170/VPP response, as well as N300 (visual cognition/knowledge) and N400 (semantic 
memory/knowledge) responses [61]. In consideration of these results, ERPs are an excellent methodology for examining processes related to mental imagery.

\section{Neuromodulation for the Creative Brain}

Whatever possibilities exist for creativity training, at least some features of creativity could stand to benefit from the addition of neuromodulation using transcranial direct current stimulation (tDCS). tDCS involves a low-cost, portable device that non-invasively delivers a safe, low electrical current to the head for the purpose of increasing (anodal electrode $[+]$ ) or decreasing (cathodal [-]) excitability in the cortical regions underlying the electrodes [62]. tDCS is a promising tool for improving cognitive, perceptual, and behavioral functions [63], with several accounts suggesting that it may also be relevant for improving creative thinking.

For example, one study found that cathodal stimulation of the left inferior frontal gyrus (IFG) - a region of the fronto-parietal control network implicated in inhibitory functions [64] in combination with anodal stimulation of the right IFG, improved performance on a verbal DT task [65]. Reversing polarity (anode-left/cathode-right) did not affect creative production, and neither did moving the cathode from the left IFG to the left orbito-frontal cortex (OFC), nor moving the anode from right IFG to right OFC. If only one of the electrodes had been doing the work of boosting creativity, it would be expected that one of these last two conditions would have been effective, so the authors suggest that a rebalancing of hemispheric control was at work. While it is possible that the action of the OFC electrodes serves as a confound, this seems like a reasonable interpretation.

Cathodal tDCS to the left PFC has also been found to increase cognitive flexibility when generating uncommon uses for objects [31]. More recently, anodal tDCS was used over the left frontopolar region to increase the semantic distance (arguably, a form of creativity) between 
pairs of words in a verbal analogy task [66]. Although anodal tDCS is thought to increase activity in the area, and increased frontal activity is not always a marker of a creative state, at least in this task anodal stimulation was effective. In fact, a prior fMRI study identified activity in this area as being reflective of verbal creativity [67]. Certainly, there is much to be learned about the interplay between brain regions serving creative functions, and the differential impact of neighboring frontal regions in particular [68].

While tDCS is increasingly popular, it is not the only type of electrical stimulation that has been used in creativity research. Transcranial alternating current stimulation (tACS), which delivers current at specific frequencies (as opposed to a constant current, in tDCS), has been shown to improve performance on a divergent thinking task when delivered to frontal regions at $10 \mathrm{~Hz}$ [69]. No such effect was found when using $40 \mathrm{~Hz}$ stimulation, strengthening the argument for the role of increased alpha in creativity. In a more recent 4-experiment study, delivering 10 $\mathrm{Hz}$ tACS to the right temporal lobe was found to increase CRA task performance when words shared misleading (ultimately wrong) associations, and increase the remoteness of AUT responses (but not creativity or cleverness) [70]. Additionally, this study found increased right temporal alpha for correct responses in a misleading-associates CRA task, and when generating more, as opposed to less remote AUT responses. Considering the combined results of these four experiments, the authors suggest that the role of alpha activity is to suppress obvious associations, allowing more remote associations to take form. The ability to specifically target frequency ranges of interest in creativity research is an enticing path for future investigations.

\section{Conclusions}

Overall, given the well-established potential for EEG to illuminate complex and transient creativity-related neural processes, further explorations of the creative brain should incorporate 
its use in a highly varied set of circumstances. Here we highlighted some of the most common tasks used to assess creative thinking, though there are others, including assessments of analogical thinking [71], and real-world creative achievement [72, 73]. Generally speaking, this review suggests that increased alpha activity is a common marker of various creative processes, and that more creative people tend to have more of it [17]. We have also shown that other EEG bands, including theta $[26,40]$ and gamma $[38,45,58]$ play a role in creative cognition. A more focused pursuit of understanding regarding these frequency bands may therefore be a fruitful path for future research. The inclusion of neuromodulation methods, such as tDCS and tACS, for potential guided improvement in creative cognition is a wide-open area, and may be of great interest to creators of all kinds. 


\section{Acknowledgements}

This research did not receive any specific grant funding from funding agencies in the public, commercial, or non-for-profit sectors. 


\section{References}

[1] Teplan, M. (2002). Fundamentals of EEG measurement. Measurement Science Review, 2(2), $1-11$.

**[2] Cohen, M. X. (2017). Where does EEG come from and what does it mean? Trends in Neurosciences, 40(4), 208-218.

This paper provides a thorough review of the neural circuits responsible for producing various EEG features linked to functions such as cognition, movement, and emotion, in addition to descriptions of the analytical methods used to decode EEG signals.

[3] Cooper, R., Winter, A. L., Crow, H. J., \& Walter, W. G. (1965). Comparison of subcortical, cortical and scalp activity using chronically indwelling electrodes in man. Electroencephalography and Clinical Neurophysiology, 18(3), 217-228.

[4] Klimesch, W., Sauseng, P., \& Hanslmayr, S. (2007). EEG alpha oscillations: the inhibitiontiming hypothesis. Brain Research Reviews, 53(1), 63-88.

[5] Ray, W. J., \& Cole, H. W. (1985). EEG alpha activity reflects attentional demands, and beta activity reflects emotional and cognitive processes. Science, 228(4700), 750-752.

[6] Tallon-Baudry, C., \& Bertrand, O. (1999). Oscillatory gamma activity in humans and its role in object representation. Trends in Cognitive Sciences, 3(4), 151-162.

[7] Luck, S. J. (2014). An introduction to the event-related potential technique. Cambridge, MA: MIT press.

[8] Lijffijt, M., Lane, S. D., Meier, S. L., Boutros, N. N., Burroughs, S., Steinberg, J. L., Moeller, G., \& Swann, A. C. (2009). P50, N100, and P200 sensory gating: relationships with behavioral inhibition, attention, and working memory. Psychophysiology, 46(5), 10591068. 
[9] Sur, S., \& Sinha, V. K. (2009). Event-related potential: An overview. Industrial Psychiatry Journal, 18(1), 70 .

[10] Folstein, J. R., \& Van Petten, C. (2008). Influence of cognitive control and mismatch on the N2 component of the ERP: a review. Psychophysiology, 45(1), 152-170.

[11] Polich, J. (2007). Updating P300: an integrative theory of P3a and P3b. Clinical Neurophysiology, 118(10), 2128-2148.

[12] Kutas, M., \& Federmeier, K. D. (2011). Thirty years and counting: finding meaning in the N400 component of the event-related brain potential (ERP). Annual Review of Psychology, 62, 621-647.

[13] Martindale, C., \& Hasenfus, N. (1978). EEG differences as a function of creativity, stage of the creative process, and effort to be original. Biological Psychology, 6(3), 157-167.

[14] Martindale, C., \& Mines, D. (1975). Creativity and cortical activation during creative, intellectual and EEG feedback tasks. Biological Psychology, 3(2), 91-100.

[15] Sternberg, R. J., \& Lubart, T. I. (1996). Investing in creativity. American psychologist, $51(7), 677$.

[16] Guilford, J. P. (1950). Creativity. American Psychologist, 5, 444-454.

** [17] Benedek, M. (2018). The neuroscience of creative idea generation. In Z. Kapoula, J. Renoult, E. Volle, \& M. Andreatta (Eds), Exploring transdisciplinarity in art and sciences (pp. 31-48). Springer Nature, Cham.

A comprehensive review of the key findings in the field of the neuroscience of creativity, including research using EEG methodology, reporting a robust relationship between alpha-band power and creative cognition. 
[18] Fink, A., \& Benedek, M. (2014). EEG alpha power and creative ideation. Neuroscience \& Biobehavioral Reviews, 44, 111-123.

[19] Jauk, E., Benedek, M., \& Neubauer, A. C. (2012). Tackling creativity at its roots: Evidence for different patterns of EEG alpha activity related to convergent and divergent modes of task processing. International Journal of Psychophysiology, 84(2), 219-225.

[20] Schwab, D., Benedek, M., Papousek, I., Weiss, E. M., \& Fink, A. (2014). The time-course of EEG alpha power changes in creative ideation. Frontiers in human neuroscience, 8 , 310.

[21] Fink, A., \& Neubauer, A. C. (2008). Eysenck meets Martindale: The relationship between extraversion and originality from the neuroscientific perspective. Personality and Individual Differences, 44(1), 299-310.

* [22] Camarda, A., Salvia, E., Vidal, J., Weil, B., Poirel, N., Houde, O., Borst, G., \& Cassotti, M. (2018). Neural basis of functional fixedness during creative idea generation: An EEG study. Neuropsychologia, 18, 4-12.

The paper links individual differences in the ability to generate more remote or unusual uses for objects on the Alternative Uses Task with the ability to sustain increased alpha power in frontal and parietal regions for longer periods of time.

[23] Benedek, M., Bergner, S., Könen, T., Fink, A., \& Neubauer, A. C. (2011). EEG alpha synchronization is related to top-down processing in convergent and divergent thinking. Neuropsychologia, 49(12), 3505-3511.

[24] Ritter, S. M., Abbing, J., \& Van Schie, H. T. (2018). Eye-closure enhances creative performance on divergent and convergent creativity tasks. Frontiers in Psychology, 9, 1315. 
* [25] Boot, N., Baas, M., Mühlfeld, E., de Dreu, C. K., \& van Gaal, S. (2017). Widespread neural oscillations in the delta band dissociate rule convergence from rule divergence during creative idea generation. Neuropsychologia, 104, 8-17.

This empirical paper uses a novel approach to investigate differences in neural mechanisms between divergent and convergent thinking. Findings indicate that compared to rule convergent thinking, rule divergent thinking was associated with widespread decreases in delta band activity.

** [26] Wokke, M. E., Ridderinkhof, K. R., \& Padding, L. (2018). Creative Minds Are Out of Control: Mid Frontal Theta and Creative Thinking. bioRxiv, 370494.

This study provides empirical evidence that creativity, as assessed with providing original captions to pictures, was associated with decreased mid-frontal theta power, providing further evidence that at least some forms of creativity may be linked with lower levels of top-down control.

[27] Clayton, M. S., Yeung, N., \& Kadosh, R. C. (2015). The roles of cortical oscillations in sustained attention. Trends in Cognitive Sciences, 19(4), 188-195.

This paper provides a thorough review of the neural circuits responsible for producing various EEG features linked to functions such as cognition, movement, and emotion, in addition to descriptions of the analytical methods used to decode EEG signals.

[28] Cavanagh, J. F., \& Frank, M. J. (2014). Frontal theta as a mechanism for cognitive control. Trends in Cognitive Sciences, 18(8), 414-421.

* [29] Zabelina, D. L., \& Ganis, G. (in press). Creativity and cognitive control: Behavioral and ERP evidence that divergent thinking, but not real-life creative achievement, relates to better cognitive control. Neuropsychologia.

This paper shows that cognitive control may differ depending on the form of creativity, as 
divergent thinking was linked with higher levels of cognitive control, as indicated by a larger N2 differences between the frequent and the rare targets on the Oddball task, whereas real-life creative achievement showed no association with cognitive control.

[30] Chrysikou, E. G., Hamilton, R. H., Coslett, H. B., Datta, A., Bikson, M., \& ThompsonSchill, S. L. (2013). Noninvasive transcranial direct current stimulation over the left prefrontal cortex facilitates cognitive flexibility in tool use. Cognitive Neuroscience, 4(2), 81-89.

[31] Zabelina, D. L., O’Leary, D., Pornpattananangkul, N., Nusslock, R., \& Beeman, M. (2015). Creativity and sensory gating indexed by the P50: Selective versus leaky sensory gating in divergent thinkers and creative achievers. Neuropsychologia, 69, 77-84.

[32] Zabelina, D. L., \& Andrews-Hanna, J. R. (2016). Dynamic network interactions supporting internally-oriented cognition. Current Opinion in Neurobiology, 40, 86-93.

[33] Mednick, S. (1962). The associative basis of the creative process. Psychological review, 69(3), 220-232.

[34] Bowden, E. M., \& Jung-Beeman, M. (2003). Normative data for 144 compound remote associate problems. Behavior Research Methods, Instruments, \& Computers, 35(4), 634639.

[35] Kounios, J., \& Beeman, M. (2014). The cognitive neuroscience of insight. Annual Review of Psychology, 65, 71-93.

[36] Rothmaler, K., Nigbur, R., \& Ivanova, G. (2017). New insights into insight: Neurophysiological correlates of the difference between the intrinsic "aha" and the extrinsic “oh yes" moment. Neuropsychologia, 95, 204-214. 
[37] Gebuis, T., Gevers, W., \& Kadosh, R. C. (2014). Topographic representation of high-level cognition: numerosity or sensory processing? Trends in Cognitive Sciences, 18(1), 1-3.

[38] Jung-Beeman M, Bowden EM, Haberman J, Frymiare JL, Arambel-Liu S, et al. (2004).

Neural activity when people solve verbal problems with insight. PLoS Biology, 2(4), e97.

[39] Herrmann, C. S., Munk, M. H., \& Engel, A. K. (2004). Cognitive functions of gamma-band activity: Memory match and utilization. Trends in Cognitive Sciences, 8(8), 347-355.

** [40] Erickson, B., Truelove-Hill, M., Oh, Y., Anderson, J., Zhang, F. Z., \& Kounios, J. (2018). Resting-state brain oscillations predict trait-like cognitive styles. Neuropsychologia, 120, 1-8.

Resting-state brain activity differentiates people's tendency to solve problems via insight or via analysis, with analytic solvers showing greater frontal beta-band activity, and insightful solvers showing more left parietal bet and left-temporal theta- and alpha-band activity.

[41] Solomon, E. A., Kragel, J. E., Sperling, M. R., Sharan, A., Worrell, G., Kucewicz, M., ... \& Jobst, B. C. (2017). Widespread theta synchrony and high-frequency desynchronization underlies enhanced cognition. Nature Communications, 8(1), 1704.

[42] Gabora, L. (in press). The neural basis and evolution of divergent and convergent thought. In O. Vartanian \& R. Jung (Eds), The Cambridge handbook of the neuroscience of creativity (pp. 58-70). Cambridge MA: Cambridge University Press.

[43] Jung-Beeman, M. (2005). Coarseness of coding and neural microcircuitry. Trends in Cognitive Sciences, 11(9), 512-518.

[44] Kounios, J., Frymiare, J. L., Bowden, E. M., Fleck, J. I., Subramaniam, K., Parrish, T. B., \& Jung-Beeman, M. (2006). The prepared mind: Neural activity prior to problem 
presentation predicts subsequent solution by sudden insight. Psychological Science, 17(10), 882-890.

[45] Berkovich-Ohana, A., Glicksohn, J., Ben-Soussan, T. D., \& Goldstein, A. (2017). Creativity is enhanced by long-term mindfulness training and is negatively correlated with trait default-mode-related low-gamma inter-hemispheric connectivity. Mindfulness, 8(3), 717 727.

[46] Margulis, E. H. (2017). Neuroscience: How music meets mind. Nature, 546(7658), 351.

[47] Simchy-Gross, R., \& Margulis, E. H. (2018). The sound-to-music illusion: Repetition can musicalize nonspeech sounds. Music \& Science, 1, 2059204317731992.

[48] Sanyal, S., Banerjee, A., Mukherjee, S., Gutherathakurata, T., Sengupta, R., \& Ghosh, D. (2016). Musical improvisation and brain correlates: An EEG based neurocognitive study using Hindustani music. Journal of Biomusical Engineering, 4(2), 119.

* [49] Goldman, A., Jackson, T., \& Sajda, P. (2018). Improvisation experience predicts how musicians categorize musical structures. Psychology of Music, 0305735618779444.

This empirical study shows that improvisationally (compared to classically) trained musicians show more discriminable N2c and P3b ERPs in response to deviant cords, suggesting that improvisationally trained musicians may have a greater sense of violation of musical expectation, likely indicating an effect of improvisational training on the brain. *[50] Przysinda, E., Zeng, T., Maves, K., Arkin, C., \& Loui, P. (2017). Jazz musicians reveal role of expectancy in human creativity. Brain and Cognition, 119, 45-53. An empirical study showing that jazz musicians prefer unexpected chord progressions, with unexpected stimuli eliciting larger early and mid-latency ERP responses (ERAN and P3b), followed by smaller long-latency responses (Late Positivity Potential) in jazz (compare to non- 
jazz) musicians.

** [51] Lopata, J. A., Nowicki, E. A., \& Joanisse, M. F. (2017). Creativity as a distinct trainable mental state: An EEG study of musical improvisation. Neuropsychologia, 99, 246-258.

The study reveals the effects of formal musical training on the brain. Musicians trained in musical improvisation showed increased frontal alpha-band activity during improvisation, compared to rote playback. Alpha activity was also greatest for the improvisations judged as more creative by independent raters.

[52] Benedek, M., Borovnjak, B., Neubauer, A. C., \& Kruse-Weber, S. (2014). Creativity and personality in classical, jazz and folk musicians. Personality and Individual Differences, 63, 117-121.

[53] Pressing, J. (1988). Improvisation: Methods and models. In J.A. Sloboda (Ed.), Generative processes in music (pp. 129-178). Oxford University Press: Oxford, UK.

[54] Pearson, J. (2014). New directions in mental-imagery research: the binocular-rivalry technique and decoding fMRI patterns. Current Directions in Psychological Science, 23(3), 178-183.

[55] Sparing, R., Mottaghy, F. M., Ganis, G., Thompson, W. L., Töpper, R., Kosslyn, S. M., \& Pascual-Leone, A. (2002). Visual cortex excitability increases during visual mental imagery - a TMS study in healthy human subjects. Brain Research, 938(1-2), 92-97.

[56] Pidgeon, L. M., Grealy, M., Duffy, A. H., Hay, L., McTeague, C., Vuletic, T., ... \& Gilbert, S. J. (2016). Functional neuroimaging of visual creativity: a systematic review and metaanalysis. Brain and Behavior, 6, 1-26, e00540. 
** [57] Rominger, C., Papousek, I., Perchtold, C. M., Weber, B., Weiss, E. M., \& Fink, A. (2018). The creative brain in the figural domain: Distinct patterns of EEG alpha power during idea generation and idea elaboration. Neuropsychologia 18, Part A, 13-19.

This empirical paper provides evidence for decreased alpha band activity in the parietal and occipital regions during idea generation in a visual picture completion task, but an increased alpha band activity during the elaboration phase.

[58] Sviderskaya, N. E., Taratynova, G. V., \& Kozhedub, R. G. (2006). The effects of the experience of forming visual images on the spatial organization of the EEG. Neuroscience and Behavioral Physiology, 36(9), 941-949.

[59] Ganis, G., \& Schendan, H. E. (2008). Visual mental imagery and perception produce opposite adaptation effects on early brain potentials. Neuroimage, 42(4), 1714-1727.

[60] Joyce, C., \& Rossion, B. (2005). The face-sensitive N170 and VPP components manifest the same brain processes: the effect of reference electrode site. Clinical Neurophysiology, 116(11), 2613-2631.

[61] Schendan, H. E., \& Ganis, G. (2012). Electrophysiological potentials reveal cortical mechanisms for mental imagery, mental simulation, and grounded (embodied) cognition. Frontiers in Psychology, 3, 329.

[62] Antal, A., I. Alekseichuk, M. Bikson, J. Brockmöller, A.R. Brunoni, R. Chen, ... \& Paulus, W. (2017). Low intensity transcranial electric stimulation: Safety, ethical, legal regulatory and application guidelines. Clinical Neurophysiology, 128, 1774-1809.

[63] Nitsche, M.A., L.G. Cohen, E.M. Wassermann, A. Priori, N. Lang, A. Antal, W. Paulus, F. Hummel, P.S. Boggio, F. Fregni, and A. Pascual-Leone (2008). Transcranial direct current stimulation: State of the art 2008. Brain Stimulation, 1: 206-223. 
[64] Seeley, W. W., Matthews, B. R., Crawford, R. K., Gorno-Tempini, M. L., Foti, D., Mackenzie, I. R., \& Miller, B. L. (2007). Unravelling Boléro: progressive aphasia, transmodal creativity and the right posterior neocortex. Brain, 131(1), 39-49.

[65] Mayseless, N., \& Shamay-Tsoory, S. G. (2015). Enhancing verbal creativity: Modulating creativity by altering the balance between right and left inferior frontal gyrus with tDCS. Neuroscience, 291, 167-176.

[66] Green, A. E., Spiegel, K. A., Giangrande, E. J., Weinberger, A. B., Gallagher, N. M., \& Turkeltaub, P. E. (2016). Thinking cap plus thinking zap: tDCS of frontopolar cortex improves creative analogical reasoning and facilitates conscious augmentation of state creativity in verb generation. Cerebral Cortex, 27(4), 2628-2639.

[67] Green, A. E., Cohen, M. S., Raab, H. A., Yedibalian, C. G., \& Gray, J. R. (2015). Frontopolar activity and connectivity support dynamic conscious augmentation of creative state. Human Brain Mapping, 36(3), 923-934.

** [68] Weinberger, A. B., Green, A. E., \& Chrysikou, E. G. (2017). Using transcranial direct current stimulation to enhance creative cognition: Interactions between task, polarity, and stimulation site. Frontiers in Human Neuroscience, 11, 246.

A synthesis of tDCS literature for enhancement of creative cognition, showing that idea generation benefits from cathodal stimulation over the left inferior frontotemporal cortex, while idea selection benefits from anodal tDCS over left dorsolateral prefrontal cortex and frontopolar cortex. The discussion includes suggestions for future research in stimulating creative thinking. [69] Lustenberger, C., Boyle, M. R., Foulser, A. A., Mellin, J. M., \& Fröhlich, F. (2015). Functional role of frontal alpha oscillations in creativity. Cortex, 67, 74-82. 
** [70] Luft, C. D. B., Zioga, I., Thompson, N. M., Banissy, M. J., \& Bhattacharya, J. (2018). Right temporal alpha oscillations as a neural mechanism for inhibiting obvious associations. Proceedings of the National Academy of Sciences, 201811465.

This study demonstrated the effects of alpha-targeted $(10 \mathrm{~Hz})$ tACS on creativity. Stimulation was also administered at $40 \mathrm{~Hz}$ (gamma band), allowing researchers to demonstrate that electrical stimulation generally (regardless of frequency) was not responsible for improved CRA task performance when words shared misleading associations, greater remoteness of uses generated in an AUT, and increased alpha power at the site of stimulation (left temporal).

[71] Green, A. E., Kraemer, D. J., Fugelsang, J. A., Gray, J. R., \& Dunbar, K. N. (2012). Neural correlates of creativity in analogical reasoning. Journal of Experimental Psychology: Learning, Memory, and Cognition, 38(2), 264.

[72] Diedrich, J., Jauk, E., Silvia, P. J., Gredlein, J. M., Neubauer, A. C., \& Benedek, M. (2017). Assessment of real-life creativity: The Inventory of Creative Activities and Achievements (ICAA). Psychology of Aesthetics, Creativity, and the Arts, 12(3), 304-316.

[73] Carson, S. H., Peterson, J. B., \& Higgins, D. M. (2005). Reliability, validity, and factor structure of the creative achievement questionnaire. Creativity Research Journal, 17(1), $37-50$.

[74] Sadaghiani, S., \& Kleinschmidt, A. (2016). Brain networks and $\alpha$-oscillations: Structural and functional foundations of cognitive control. Trends in Cognitive Sciences, 20(11), 805-817. 
Figure 1. The upper portion of the figure shows a time-frequency decomposition EEG signal power across a portion of the overall frequency spectrum $(3-30 \mathrm{~Hz})$. This recording was taken at electrode site $\mathrm{Oz}$, located on the midline of the occipital [visual] cortex. Red color indicates higher power in a given frequency range (y-axis, to the left), and blue color indicates lower power. The $\mathrm{x}$-axis is the time domain. Here, power in the alpha range is higher for an attentional shift, indicating a suppression of visual information/attention. The lower portion of the figure shows a standard graph of EEG power, with a downward left-to-right slope (greater signal amplitude for lower frequencies). Also visible is the increase in alpha power $(\sim 10 \mathrm{~Hz}) 3 \mathrm{~seconds}$ after time 0 (event) for the graph to the right. Source: figure adapted with permission from [74].

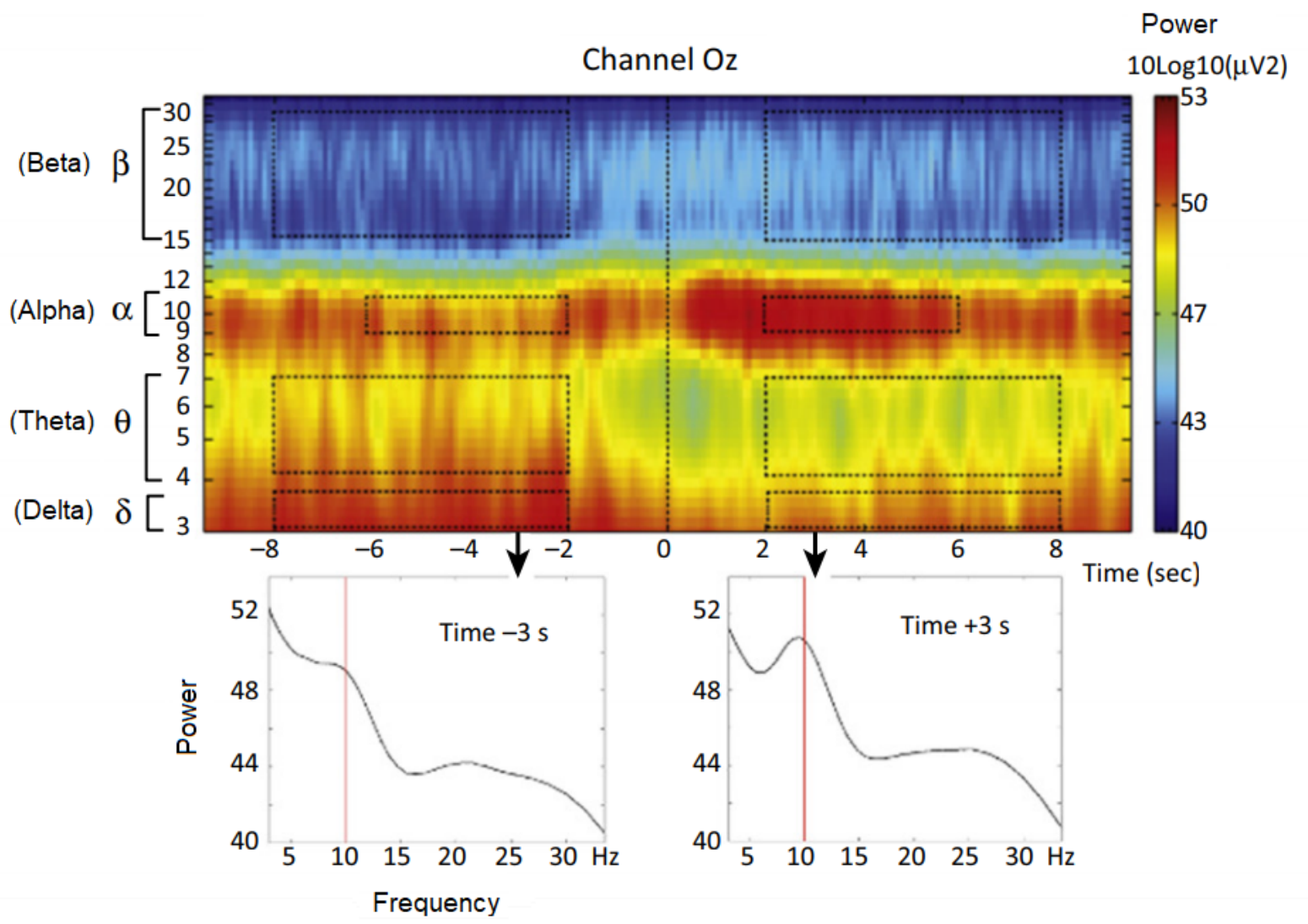


Figure 2. Individual difference studies generally show that increased EEG alpha power is particularly sensitive to various creativity-related demands involved in divergent thinking, although other EEG bands have also been shown to play a role in creative cognition. (a) Displayed here are differences in alpha between individuals with higher and lower originality scores on the Alternative Uses Task, showing that higher original people showed stronger increases in alpha in the right than in the left hemisphere, while lower original people showed no hemispheric differences with respect to alpha power. Blue regions reflect greater alpha power relative to rest. AF: anteriofrontal; F: frontal; FC: frontocentral; CT: centrotemporal; $\mathrm{CP}$ : centroparietal; PT: parietotemporal; PO: parietooccipital. Source: figure adapted with permission from [18]; (b) Involvement of theta band activity in divergent thinking, showing decreased frontal theta activity in people who provided more creative captions for various unusual scenes. Source: figure adapted with permission from [**26].

(a)

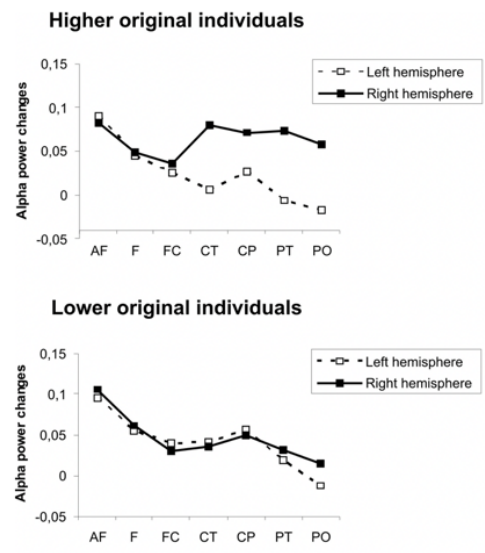

(b)

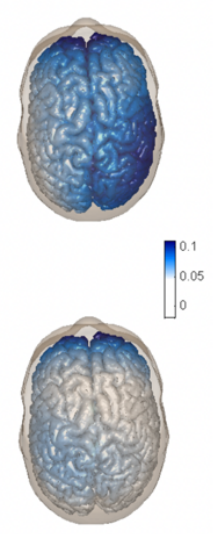

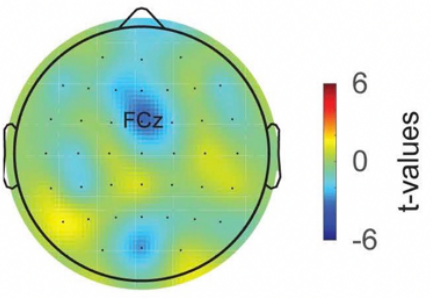

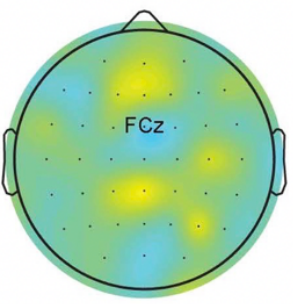

0-500 ms after button press 


\section{Figure 3.}

When people solve Compound Remote Associate (CRA) problems, they can generally report on whether the solution was reached via insight or via analysis. Neural activity recorded during solution of CRA problems suggest that these two types of cognitive strategies rely on distinct cognitive mechanisms. (a) an increase in alpha-band activity $(10 \mathrm{~Hz})$ is seen over the occipital cortex moments just prior to solutions reached via insight (versus via analysis), immediately followed by a burst of high frequency gamma-band activity originating in the right temporal lobe. Source: figure adapted with permission from [38]. (b) Resting-state EEG predicts people's tendency to solve Compound Remote Associate (CRA) problems with insight or with analysis. Beta band activity over left parietal (insight $>$ analytic, in red), and beta band activity over midline frontal cortex (analytic>insight, in blue) predicts solution type weeks in advance. Alpha and theta band clusters extending from left anterior to posterior temporal cortex spatially overlap in predicting insight solutions (insight $>$ analytic, in red). Source: figures adapted with permission from Source: figure adapted with permission from [40].

(a)

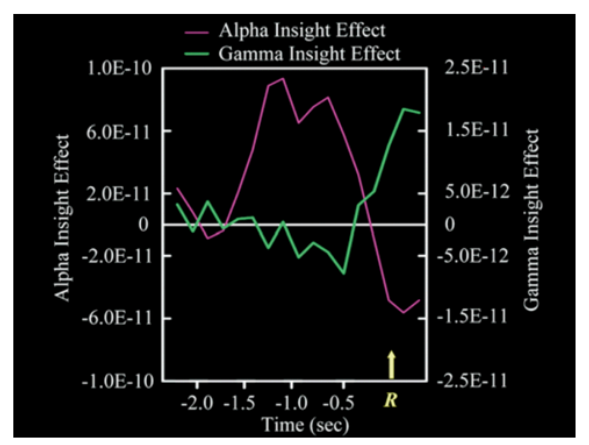

(b)

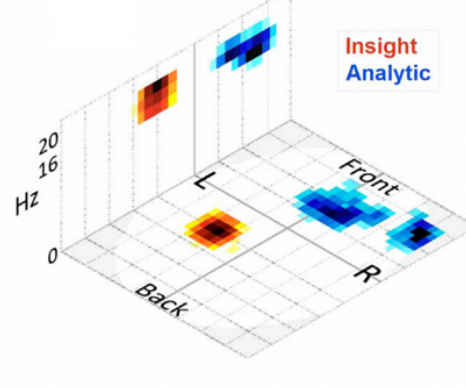

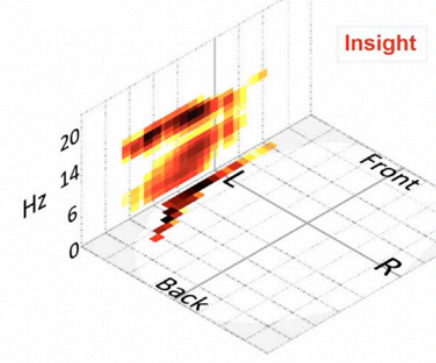




\section{Figure 4 .}

Improvisational jazz is shown to be associated with alpha band power. (a) Skilled musicians with training in musical improvisation (compared to musicians with no formal improvisation training) show increased frontal alpha-band activity during improvisation compared to during rote playback. FITI = formal improvisation training individuals. (b) The quality of the improvised pieces is associated with higher alpha power in musicians with formal training. Source: figure adapted with permission from [51].

\section{(a)}

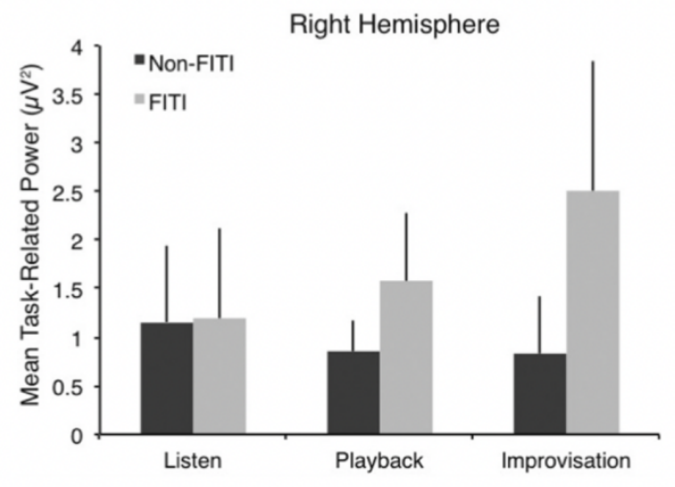

(b)

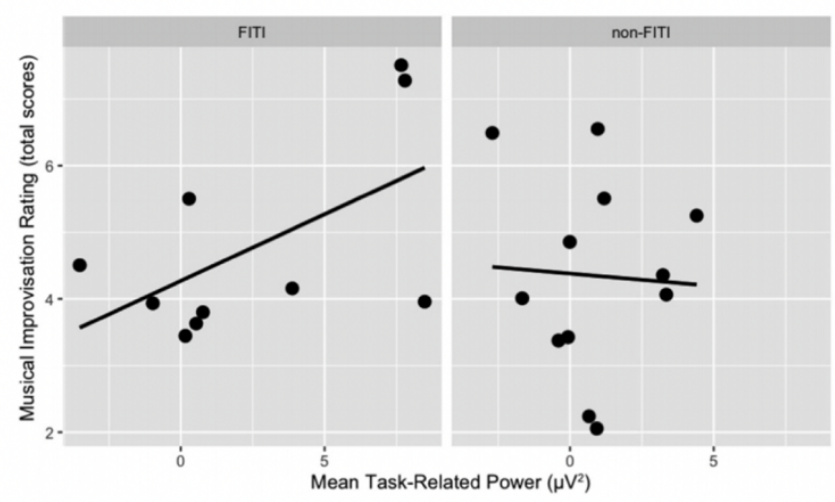

